\title{
Weak contractive inequalities and compatible mixed monotone random operators in ordered metric spaces
}

\author{
N Hussain', A Latif ${ }^{*}$ and N Shafqat ${ }^{2}$
}

"Correspondence: alatif@kau.edu.sa 1 Department of Mathematics, King Abdulaziz University, P.O. Box 80203, Jeddah, 21589, Saudi Arabia Full list of author information is available at the end of the article

\begin{abstract}
The aim of this work is to define the notion of compatible random operators in a partially ordered metric space and prove some coupled random coincidence theorems for a pair of compatible mixed monotone random operators satisfying $(\phi, \varphi)$-weak contractive conditions. These results present random versions and extensions of recent results of Ćirić and Lakshmikantham (Stoch. Anal. Appl. 27:1246-1259, 2009), Choudhury and Kundu (Nonlinear Anal. 73:2524-2531, 2010), Alotaibi and Alsulami (Fixed Point Theory Appl. 2011:44, 2011) and many others.
\end{abstract}

\section{Introduction}

Random coincidence point theorems are stochastic generalizations of classical coincidence point theorems. Some random fixed point theorems play an important role in the theory of random differential and random integral equations (see [1, 2]). Random fixed point theorems for contractive mappings on separable complete metric spaces have been proved by several authors [3-8]. Sehgal and Singh [9] have proved different stochastic versions of the well-known Schauder fixed point theorem. Fixed point theorems for monotone operators in ordered Banach spaces have been investigated and have found various applications in differential and integral equations (see [10-12] and references therein). Fixed point theorems for mixed monotone mappings in partially ordered metric spaces are of great importance and have been utilized for matrix equations, ordinary differential equations, and for the existence and uniqueness of solutions for some boundary value problems (see [13-19]).

Recently Ćirić and Lakshmikantham [20] and Zhu and Xiao [21] proved some coupled random fixed point and coupled random coincidence results in partially ordered complete metric spaces. The purpose of this article is to improve these results for a pair of compatible mixed monotone random mappings $F: \Omega \times(X \times X) \rightarrow X$ and $g: \Omega \times X \rightarrow X$, where $F$ and $g$ satisfy the $(\phi, \varphi)$-weak contractive conditions. Presented results are also the extensions and improvements of the corresponding results in [22-24] and many others.

\section{Preliminaries}

Recall that if $(X, \leq)$ is a partially ordered set and $F: X \rightarrow X$ is such that for $x, y \in X$, $x \leq y$ implies $F(x) \leq F(y)$, then a mapping $F$ is said to be non-decreasing. Similarly, a

(C) 2012 Hussain et al.; licensee Springer. This is an Open Access article distributed under the terms of the Creative Commons Attribution License (http://creativecommons.org/licenses/by/2.0), which permits unrestricted use, distribution, and reproduction in any medium, provided the original work is properly cited. 
non-increasing map may be defined. Bhaskar and Lakshmikantham [25] introduced the following notions of a mixed monotone mapping and a coupled fixed point.

Definition 2.1 ([25]) Let $(X, \leq)$ be a partially ordered set and $F: X \times X \rightarrow X$. The mapping $F$ is said to have the mixed monotone property if $F$ is monotone non-decreasing in its first argument and is monotone non-increasing in its second argument, that is, for any $x, y \in X$,

$$
x_{1}, x_{2} \in X ; \quad x_{1} \leq x_{2} \quad \Rightarrow \quad F\left(x_{1}, y\right) \leq F\left(x_{2}, y\right)
$$

and

$$
y_{1}, y_{2} \in X ; \quad y_{1} \leq y_{2} \quad \Rightarrow \quad F\left(x, y_{1}\right) \geq F\left(x, y_{2}\right) .
$$

Definition $2.2([25])$ An element $(x, y) \in X \times X$ is called a coupled fixed point of the mapping $F: X \times X \rightarrow X$ if

$$
F(x, y)=x, \quad F(y, x)=y .
$$

The concept of the mixed monotone property is generalized in [24].

Definition 2.3 ([24]) Let $(X, \leq)$ be a partially ordered set and $F: X \times X \rightarrow X$ and $g: X \rightarrow X$. The mapping $F$ is said to have the mixed $g$-monotone property if $F$ is monotone $g$-non-decreasing in its first argument and is monotone $g$-non-increasing in its second argument, that is, for any $x, y \in X$,

$$
x_{1}, x_{2} \in X ; \quad g\left(x_{1}\right) \leq g\left(x_{2}\right) \quad \Rightarrow \quad F\left(x_{1}, y\right) \leq F\left(x_{2}, y\right)
$$

and

$$
y_{1}, y_{2} \in X ; \quad g\left(y_{1}\right) \leq g\left(y_{2}\right) \quad \Rightarrow \quad F\left(x, y_{1}\right) \geq F\left(x, y_{2}\right) .
$$

Definition 2.4 An element $(x, y) \in X \times X$ is called a coupled fixed point of the mapping $F: X \times X \rightarrow X$ and $g: X \rightarrow X$ if

$$
F(x, y)=g(x), \quad F(y, x)=g(y) .
$$

Definition 2.5 The mappings $F$ and $g$, where $F: X \times X \rightarrow X$ and $g: X \rightarrow X$, are said to be compatible if

$$
\lim _{n \rightarrow \infty} d\left(g\left(F\left(x_{n}, y_{n}\right)\right), F\left(g\left(x_{n}\right), g\left(y_{n}\right)\right)\right)=0
$$

and

$$
\lim _{n \rightarrow \infty} d\left(g\left(F\left(y_{n}, x_{n}\right)\right), F\left(g\left(y_{n}\right), g\left(x_{n}\right)\right)\right)=0,
$$

whenever $\left\{x_{n}\right\},\left\{y_{n}\right\}$ are sequences in $X$ such that $\lim _{n \rightarrow \infty} F\left(x_{n}, y_{n}\right)=\lim _{n \rightarrow \infty} g\left(x_{n}\right)=x$ and $\lim _{n \rightarrow \infty} F\left(y_{n}, x_{n}\right)=\lim _{n \rightarrow \infty} g\left(y_{n}\right)=y$ for all $x, y \in X$ are satisfied. 
Using the concept of compatible maps and the mixed $g$-monotone property, Choudhury and Kundu [23] proved the following theorem.

Theorem 2.6 Let $(X, \leq)$ be a partially ordered set, and let there be a metric $d$ on $X$ such that $(X, d)$ is a complete metric space. Let $\varphi:[0, \infty) \rightarrow[0, \infty)$ be such that $\varphi(t)<t$ and $\lim i t_{r \rightarrow t^{+}} \varphi(r)<t$ for all $t>0$. Let $F: X \times X \rightarrow X$ and $g: X \rightarrow X$ be two mappings such that $F$ has the mixed g-monotone property and satisfy

$$
d(F(x, y), F(u, v)) \leq \varphi\left(\frac{d(g x, g u)+d(g y, g v)}{2}\right)
$$

for all $x, y, u, v \in X$, for which $g x \leq$ gu and $g y \geq g v$. Let $F(X \times X) \subseteq g(X)$, $g$ be continuous and monotone increasing and $F$ and $g$ be compatible mappings. Also, suppose either

(a) $F$ is continuous or

(b) $X$ has the following properties:

(i) if a non-decreasing sequence $\left\{x_{n}\right\} \rightarrow x$, then $x_{n} \leq x$ for all $n \geq 0$,

(ii) if a non-increasing sequence $\left\{y_{n}\right\} \rightarrow y$, then $y_{n} \geq y$ for all $n \geq 0$.

If there exist $x_{0}, y_{0} \in X$, such that $g\left(x_{0}\right) \leq F\left(x_{0}, y_{0}\right)$ and $g\left(y_{0}\right) \geq F\left(y_{0}, x_{0}\right)$, then there exist $x, y \in X$ such that $g(x)=F(x, y)$ and $g(y)=F(y, x)$, that is, $F$ and $g$ have a coupled coincidence point in $X$.

As in [17], let $\Phi$ denote all functions $\phi:[0, \infty) \rightarrow[0, \infty)$ which satisfy

1. $\phi$ is continuous and non-decreasing,

2. $\phi(t)=0$ if and only if $t=0$,

3. $\phi(t+s) \leq \phi(t)+\phi(s), \forall t, s \in[0, \infty)$,

and let $\Psi$ denote all the functions $\psi:[0, \infty) \rightarrow(0, \infty)$ which satisfy $\lim _{t \rightarrow r} \psi(t)>0$ for all $r>0$ and $\lim _{t \rightarrow 0^{+}} \psi(t)=0$.

Alotaibi and Alsulami in [22] proved the following coupled coincidence result for monotone operators in partially ordered metric spaces.

Theorem 2.7 Let $(X, \leq)$ be a partially ordered set and suppose there is a metric $d$ on $X$ such that $(X, d)$ is a complete metric space. Let $F: X \times X \rightarrow X$ be a mapping having the mixed $g$-monotone property on $X$ such that there exist two elements $x_{0}, y_{0} \in X$ with

$$
g x_{0} \leq F\left(x_{0}, y_{0}\right) \text { and } g y_{0} \geq F\left(y_{0}, x_{0}\right) \text {. }
$$

Suppose there exist $\phi \in \Phi$ and $\varphi \in \Psi$ such that

$$
\phi(d(F(x, y), F(u, v))) \leq \frac{1}{2} \phi(d(g x, g u)+d(g y, g v))-\psi\left(\frac{d(g x, g u)+d(g y, g v)}{2}\right)
$$

for all $x, y, u, v \in X$ with $g x \geq g u$ and $g y \leq g v$. Suppose $F(X \times X) \subseteq g(X), g$ is continuous and compatible with $F$ and also suppose either

(a) F is continuous or

(b) $X$ has the following property:

(i) if a non-decreasing sequence $\left\{x_{n}\right\} \rightarrow x$, then $x_{n} \leq x$ for all $n$,

(ii) if a non-increasing sequence $\left\{y_{n}\right\} \rightarrow y$, then $y \leq y_{n}$ for all $n$. 
Then there exists $x, y \in X$ such that

$$
g x=F(x, y) \quad \text { and } \quad g y=F(y, x)
$$

that is, $F$ and $g$ have a coupled coincidence point in $X$.

\section{Main results}

Let $(\Omega, \Sigma)$ be a measurable space with $\Sigma$ being a sigma algebra of subsets of $\Omega$, and let $(X, d)$ be a metric space. A mapping $T: \Omega \rightarrow X$ is called $\Sigma$-measurable if for any open subset $U$ of $X, T^{-1}(U)=\{\omega: T(\omega) \in U\} \in \Sigma$. In what follows, when we speak of measurability, we will mean $\Sigma$-measurability. A mapping $T: \Omega \times X \rightarrow X$ is called a random operator if for any $x \in X, T(\cdot, x)$ is measurable. A measurable mapping $\zeta: \Omega \rightarrow X$ is called a random fixed point of a random function $T: \Omega \times X \rightarrow X$ if $\zeta(\omega)=T(\omega, \zeta(\omega))$ for every $\omega \in \Omega$. A measurable mapping $\zeta: \Omega \rightarrow X$ is called a random coincidence of $T: \Omega \times X \rightarrow X$ and $g: \Omega \times X \rightarrow X$ if $g(\omega, \zeta(\omega))=T(\omega, \zeta(\omega))$ for every $\omega \in \Omega$.

Definition 3.1 Let $(X, d)$ be a separable metric space and $(\Omega, \Sigma)$ be a measurable space. Then $F: \Omega \times(X \times X) \rightarrow X$ and $g: \Omega \times X \rightarrow X$ are said to be compatible random operators if

$$
\lim _{n \rightarrow \infty} d\left(g\left(\omega, F\left(\omega,\left(x_{n}, y_{n}\right)\right)\right), F\left(\omega,\left(g\left(\omega, x_{n}\right), g\left(\omega, y_{n}\right)\right)\right)\right)=0
$$

and

$$
\lim _{n \rightarrow \infty} d\left(g\left(\omega, F\left(\omega,\left(y_{n}, x_{n}\right)\right)\right), F\left(\omega,\left(g\left(\omega, y_{n}\right), g\left(\omega, x_{n}\right)\right)\right)\right)=0
$$

whenever $\left\{x_{n}\right\},\left\{y_{n}\right\}$ are sequences in $X$, such that $\lim _{n \rightarrow \infty} F\left(\omega,\left(x_{n}, y_{n}\right)\right)=\lim _{n \rightarrow \infty} g\left(\omega, x_{n}\right)=$ $x$ and $\lim _{n \rightarrow \infty} F\left(\omega,\left(y_{n}, x_{n}\right)\right)=\lim _{n \rightarrow \infty} g\left(\omega, y_{n}\right)=y$ for all $\omega \in \Omega$ and $x, y \in X$ are satisfied.

As in [23], let $\varphi:[0, \infty) \rightarrow[0, \infty)$ be such that $\varphi(t)<t$ and $\lim i t_{r \rightarrow t^{+}} \varphi(r)<t$ for all $t>0$. Now, we state our main result.

Theorem 3.2 Let $(X, \leq, d)$ be a complete separable partially ordered metric space, $(\Omega, \Sigma)$ be a measurable space, and $F: \Omega \times(X \times X) \rightarrow X$ and $g: \Omega \times X \rightarrow X$ be mappings such that

(i) $g(\omega, \cdot)$ is continuous for all $\omega \in \Omega$;

(ii) $F(\cdot, v), g(\cdot, x)$ are measurable for all $v \in X \times X$ and $x \in X$ respectively;

(iii) $F(\omega, \cdot)$ has the mixed $g(\omega, \cdot)$-monotone property for each $\omega \in \Omega$ and

$$
d(F(\omega,(x, y)), F(\omega,(u, v))) \leq \varphi\left(\frac{d(g(\omega, x), g(\omega, u))+d(g(\omega, y), g(\omega, v))}{2}\right)
$$

for all $x, y, u, v \in X$, for which $g(\omega, x) \leq g(\omega, u)$ and $g(\omega, y) \geq g(\omega, v)$ for all $\omega \in \Omega$.

Suppose $g(\omega \times X)=X$ for each $\omega \in \Omega, g$ is monotone increasing, and $F$ and $g$ are compatible random operators. Also suppose either

(a) $F(\omega, \cdot)$ is continuous for all $\omega \in \Omega$ or 
(b) X has the following property:

$$
\begin{aligned}
& \text { if a non-decreasing sequence }\left\{x_{n}\right\} \rightarrow x \text {, then } x_{n} \leq x \text { for all } n, \\
& \text { if a non-increasing sequence }\left\{y_{n}\right\} \rightarrow y \text {, then } y_{n} \geq y \text { for all } n \text {. }
\end{aligned}
$$

If there exist measurable mappings $\zeta_{0}, \eta_{0}: \Omega \rightarrow X$ such that $g\left(\omega, \zeta_{0}(\omega)\right) \leq F\left(\omega,\left(\zeta_{0}(\omega)\right.\right.$, $\left.\left.\eta_{0}(\omega)\right)\right)$ and $g\left(\omega, \eta_{0}(\omega)\right) \geq F\left(\omega,\left(\eta_{0}(\omega), \zeta_{0}(\omega)\right)\right)$ for all $\omega \in \Omega$, then there are measurable mappings $\zeta, \theta: \Omega \rightarrow X$ such that $F(\omega,(\zeta(\omega), \theta(\omega)))=g(\omega, \zeta(\omega))$ and $F(\omega,(\theta(\omega), \zeta(\omega)))=$ $g(\omega, \theta(\omega))$ for all $\omega \in \Omega$, that is, $F$ and $g$ have a coupled random coincidence point.

Proof Let $\Theta=\{\zeta: \Omega \rightarrow X\}$ be a family of measurable mappings. Define a function $h$ : $\Omega \times X \rightarrow R^{+}$as follows:

$$
h(\omega, x)=d(x, g(\omega, x)) .
$$

Since $x \rightarrow g(\omega, x)$ is continuous for all $\omega \in \Omega$, we conclude that $h(\omega, \cdot)$ is continuous for all $\omega \in \Omega$. Also, since $x \rightarrow g(\omega, x)$ is measurable for all $x \in X$, we conclude that $h(\cdot, x)$ is measurable for all $\omega \in \Omega$ (see [26], p.868). Thus, $h(\omega, x)$ is the Caratheodory function. Therefore, if $\zeta: \Omega \rightarrow X$ is a measurable mapping, then $\omega \rightarrow h(\omega, \zeta(\omega))$ is also measurable (see [27]). Also, for each $\zeta \in \Theta$, the function $\eta: \Omega \rightarrow X$ defined by $\eta(\omega)=g(\omega, \zeta(\omega))$ is measurable, that is, $\eta \in \Theta$.

Now, we shall construct two sequences of measurable mappings $\left\{\zeta_{n}\right\}$ and $\left\{\eta_{n}\right\}$ in $\Theta$, and two sequences $\left\{g\left(\omega, \zeta_{n}(\omega)\right)\right\}$ and $\left\{g\left(\omega, \eta_{n}(\omega)\right)\right\}$ in $X$ as follows. Let $\zeta_{0}, \eta_{0} \in \Theta$ be such that $g\left(\omega, \zeta_{0}(\omega)\right) \leq F\left(\omega,\left(\zeta_{0}(\omega), \eta_{0}(\omega)\right)\right)$ and $g\left(\omega, \eta_{0}(\omega)\right) \geq F\left(\omega,\left(\eta_{0}(\omega), \zeta_{0}(\omega)\right)\right)$ for all $\omega \in \Omega$. Since $F\left(\omega,\left(\zeta_{0}(\omega), \eta_{0}(\omega)\right)\right) \in X=g(\omega \times X)$ by an appropriate Filippov measurable implicit function theorem [1, 20, 28, 29], there is $\zeta_{1} \in \Theta$ such that $g\left(\omega, \zeta_{1}(\omega)\right)=$ $F\left(\omega,\left(\zeta_{0}(\omega), \eta_{0}(\omega)\right)\right)$. Similarly, as $F\left(\omega,\left(\eta_{0}(\omega), \zeta_{0}(\omega)\right)\right) \in g(\omega \times X)$, there is $\eta_{1}(\omega) \in \Theta$ such that $g\left(\omega, \eta_{1}(\omega)\right)=F\left(\omega,\left(\eta_{0}(\omega), \zeta_{0}(\omega)\right)\right)$. Now $F\left(\omega,\left(\zeta_{1}(\omega), \eta_{1}(\omega)\right)\right)$ and $F\left(\omega,\left(\eta_{1}(\omega), \zeta_{1}(\omega)\right)\right)$ are well defined. Again from $F\left(\omega,\left(\zeta_{1}(\omega), \eta_{1}(\omega)\right)\right), F\left(\omega,\left(\eta_{1}(\omega), \zeta_{1}(\omega)\right)\right) \in g(\omega \times X)$, there are $\zeta_{2}, \eta_{2} \in \Theta$ such that $g\left(\omega, \zeta_{2}(\omega)\right)=F\left(\omega,\left(\zeta_{1}(\omega), \eta_{1}(\omega)\right)\right)$ and $g\left(\omega, \eta_{2}(\omega)\right)=F\left(\omega,\left(\eta_{1}(\omega), \zeta_{1}(\omega)\right)\right)$. Continuing this process, we can construct sequences $\left\{\zeta_{n}(\omega)\right\}$ and $\left\{\eta_{n}(\omega)\right\}$ in $X$ such that

$$
\begin{aligned}
& g\left(\omega, \zeta_{n+1}(\omega)\right)=F\left(\omega,\left(\zeta_{n}(\omega), \eta_{n}(\omega)\right)\right) \quad \text { and } \\
& g\left(\omega, \eta_{n+1}(\omega)\right)=F\left(\omega,\left(\eta_{n}(\omega), \zeta_{n}(\omega)\right)\right)
\end{aligned}
$$

for all $n \geq 0$.

We shall prove that

$$
g\left(\omega, \zeta_{n}(\omega)\right) \leq g\left(\omega, \zeta_{n+1}(\omega)\right) \quad \text { for all } n \geq 0
$$

and

$$
g\left(\omega, \eta_{n}(\omega)\right) \geq g\left(\omega, \eta_{n+1}(\omega)\right) \quad \text { for all } n \geq 0 .
$$

The proof will be given by mathematical induction. Let $n=0$. By assumption we have $g\left(\omega, \zeta_{0}(\omega)\right) \leq F\left(\omega,\left(\zeta_{0}(\omega), \eta_{0}(\omega)\right)\right)$ and $g\left(\omega, \eta_{0}(\omega)\right) \geq F\left(\omega,\left(\eta_{0}(\omega), \zeta_{0}(\omega)\right)\right)$. Since $g\left(\omega, \zeta_{1}(\omega)\right)=$ 
$F\left(\omega,\left(\zeta_{0}(\omega), \eta_{0}(\omega)\right)\right)$ and $g\left(\omega, \eta_{1}(\omega)\right)=F\left(\omega,\left(\eta_{0}(\omega), \zeta_{0}(\omega)\right)\right)$, we have

$$
g\left(\omega, \zeta_{0}(\omega)\right) \leq g\left(\omega, \zeta_{1}(\omega)\right) \text { and } g\left(\omega, \eta_{0}(\omega)\right) \geq g\left(\omega, \eta_{1}(\omega)\right)
$$

Therefore, (5) and (6) hold for $n=0$.

Suppose now that (5) and (6) hold for some fixed $n \geq 0$. Then, since $g\left(\omega, \zeta_{n}(\omega)\right) \leq$ $g\left(\omega, \zeta_{n+1}(\omega)\right)$ and $g\left(\omega, \eta_{n}(\omega)\right) \geq g\left(\omega, \eta_{n+1}(\omega)\right)$ and as $F$ is monotone $g$-non-decreasing in its first argument, from (2) and (4), we have

$$
\begin{aligned}
& F\left(\omega,\left(\zeta_{n}(\omega), \eta_{n}(\omega)\right)\right) \leq F\left(\omega,\left(\zeta_{n_{+1}}(\omega), \eta_{n}(\omega)\right)\right) \quad \text { and } \\
& F\left(\omega,\left(\eta_{n+1}(\omega), \zeta_{n}(\omega)\right)\right) \leq F\left(\omega,\left(\eta_{n}(\omega), \zeta_{n}(\omega)\right)\right) .
\end{aligned}
$$

Similarly, from (3) and (4), as $g\left(\omega, \eta_{n+1}(\omega)\right) \leq g\left(\omega, \eta_{n}(\omega)\right)$ and $g\left(\omega, \zeta_{n}(\omega)\right) \leq g\left(\omega, \zeta_{n+1}(\omega)\right)$,

$$
\begin{aligned}
& F\left(\omega,\left(\zeta_{n+1}(\omega), \eta_{n+1}(\omega)\right)\right) \geq F\left(\omega,\left(\zeta_{n_{+1}}(\omega), \eta_{n}(\omega)\right)\right) \quad \text { and } \\
& F\left(\omega,\left(\eta_{n+1}(\omega), \zeta_{n}(\omega)\right)\right) \geq F\left(\omega,\left(\eta_{n+1}(\omega), \zeta_{n+1}(\omega)\right)\right) .
\end{aligned}
$$

Now from (7), (8), and (4), we get

$$
g\left(\omega, \zeta_{n+1}(\omega)\right) \leq g\left(\omega, \zeta_{n+2}(\omega)\right)
$$

and

$$
g\left(\omega, \eta_{n+1}(\omega)\right) \geq g\left(\omega, \eta_{n+2}(\omega)\right) .
$$

Thus, by mathematical induction we conclude that (5) and (6) hold for all $n \geq 0$.

Denote for each $\omega \in \Omega$

$$
\delta_{n}=d\left(g\left(\omega, \zeta_{n}(\omega)\right), g\left(\omega, \zeta_{n+1}(\omega)\right)\right)+d\left(g\left(\omega, \eta_{n}(\omega)\right), g\left(\omega, \eta_{n+1}(\omega)\right)\right)
$$

We show that

$$
\frac{\delta_{n}}{2} \leq \varphi\left(\frac{\delta_{n-1}}{2}\right) \quad \text { for all } n \geq 1
$$

Since from (5) and (6) we have $g\left(\omega, \zeta_{n-1}(\omega)\right) \leq g\left(\omega, \zeta_{n}(\omega)\right)$ and $g\left(\omega, \eta_{n-1}(\omega)\right) \geq g\left(\omega, \eta_{n}(\omega)\right)$, therefore from (4) and (1), we get

$$
\begin{aligned}
& d\left(g\left(\omega, \zeta_{n}(\omega)\right), g\left(\omega, \zeta_{n+1}(\omega)\right)\right) \\
& \quad=d\left(F\left(\omega,\left(\zeta_{n-1}(\omega), \eta_{n-1}(\omega)\right)\right), F\left(\omega,\left(\zeta_{n}(\omega), \eta_{n}(\omega)\right)\right)\right) \\
& \quad \leq \varphi\left(\frac{d\left(g\left(\omega, \zeta_{n-1}(\omega)\right), g\left(\omega, \zeta_{n}(\omega)\right)\right)+d\left(g\left(\omega, \eta_{n-1}(\omega)\right), g\left(\omega, \eta_{n}(\omega)\right)\right)}{2}\right) \\
& \quad=\varphi\left(\frac{\delta_{n-1}}{2}\right) .
\end{aligned}
$$


Similarly, from (4) and (1), as $g\left(\omega, \eta_{n}(\omega)\right) \leq g\left(\omega, \eta_{n-1}(\omega)\right)$ and $g\left(\omega, \zeta_{n}(\omega)\right) \geq g\left(\omega, \zeta_{n-1}(\omega)\right)$,

$$
\begin{aligned}
& d\left(g\left(\omega, \eta_{n+1}(\omega)\right), g\left(\omega, \eta_{n}(\omega)\right)\right) \\
& \quad=d\left(F\left(\omega,\left(\eta_{n}(\omega), \zeta_{n}(\omega)\right)\right), F\left(\omega,\left(\eta_{n-1}(\omega), \zeta_{n-1}(\omega)\right)\right)\right) \\
& \quad \leq \varphi\left(\frac{d\left(g\left(\omega, \eta_{n-1}(\omega)\right), g\left(\omega, \eta_{n}(\omega)\right)\right)+d\left(g\left(\omega, \zeta_{n-1}(\omega)\right), g\left(\omega, \zeta_{n}(\omega)\right)\right)}{2}\right) \\
& \quad=\varphi\left(\frac{\delta_{n-1}}{2}\right) .
\end{aligned}
$$

By adding (12) and (13), and dividing by 2, we obtain (11).

From (11), since $\varphi(t)<t$ for $t>0$, it follows that $\left\{\delta_{n}\right\}$ is the monotone decreasing sequence of positive reals. Therefore, there is some $\delta \geq 0$ such that

$$
\lim _{n \rightarrow \infty} \delta_{n}=\delta+
$$

We show that $\delta=0$. Suppose, to the contrary, that $\delta>0$. Taking the limit in (11) when $\delta_{n} \rightarrow \delta+$ and having in mind that we assume that $\lim _{r \rightarrow t+} \varphi(t)<t$ for all $t>0$, we have

$$
\frac{\delta}{2}=\lim _{n \rightarrow \infty} \frac{\delta_{n}}{2} \leq \lim _{n \rightarrow \infty} \varphi\left(\frac{\delta_{n-1}}{2}\right)=\lim _{\delta_{n-1} \rightarrow \delta+} \varphi\left(\frac{\delta_{n-1}}{2}\right)<\frac{\delta}{2},
$$

a contradiction. Thus, $\delta=0$.

Now we prove that for each $\omega \in \Omega,\left\{g\left(\omega, \zeta_{n}(\omega)\right)\right\}$ and $\left\{g\left(\omega, \eta_{n}(\omega)\right)\right\}$ are Cauchy sequences. Suppose, to the contrary, that at least one, $\left\{g\left(\omega, \zeta_{n}(\omega)\right)\right\}$ or $\left\{g\left(\omega, \eta_{n}(\omega)\right)\right\}$, is not a Cauchy sequence. Then there exist an $\epsilon \geq 0$ and two subsequences of positive integers $\{l(k)\},\{m(k)\}$, $m(k)>l(k) \geq k$ with

$$
\begin{aligned}
r_{k} & =d\left(g\left(\omega, \zeta_{l(k)}(\omega)\right), g\left(\omega, \zeta_{m(k)}(\omega)\right)\right)+d\left(g\left(\omega, \eta_{l(k)}(\omega)\right), g\left(\omega, \eta_{m(k)}(\omega)\right)\right) \\
& \geq \epsilon
\end{aligned}
$$

for $k \in\{1,2, \ldots\}$.

We may also assume

$$
d\left(g\left(\omega, \zeta_{l(k)}(\omega)\right), g\left(\omega, \zeta_{m(k)-1}(\omega)\right)\right)+d\left(g\left(\omega, \eta_{l(k)}(\omega)\right), g\left(\omega, \eta_{m(k)-1}(\omega)\right)\right)<\epsilon .
$$

By choosing $m(k)$ to be the smallest number exceeding $l(k)$ for which (14) holds, such $m(k)$ for which (15) holds exists, because $\delta_{n} \rightarrow 0$. From (14), (15) and by the triangle inequality, we have

$$
\begin{aligned}
\epsilon \leq & r_{k} \\
\leq & d\left(g\left(\omega, \zeta_{l(k)}(\omega)\right), g\left(\omega, \zeta_{m(k)-1}(\omega)\right)\right) \\
& +d\left(g\left(\omega, \zeta_{m(k)-1}(\omega)\right), g\left(\omega, \zeta_{m(k)}(\omega)\right)\right) \\
& +d\left(g\left(\omega, \eta_{l(k)}(\omega)\right), g\left(\omega, \eta_{m(k)-1}(\omega)\right)\right) \\
& +d\left(g\left(\omega, \eta_{m(k)-1}(\omega)\right), g\left(\omega, \eta_{m(k)}(\omega)\right)\right)
\end{aligned}
$$




$$
\begin{aligned}
= & d\left(g\left(\omega, \zeta_{l(k)}(\omega)\right), g\left(\omega, \zeta_{m(k)-1}(\omega)\right)\right) \\
& +d\left(g\left(\omega, \eta_{l(k)}(\omega)\right), g\left(\omega, \eta_{m(k)-1}(\omega)\right)\right)+\delta_{m(k)-1} \\
< & \epsilon+\delta_{m(k)-1} .
\end{aligned}
$$

Taking the limit as $k \rightarrow \infty$, we get

$$
\lim _{k \rightarrow \infty} r_{k}=\epsilon+
$$

Inequality (14) and the triangle inequality imply now

$$
\begin{aligned}
r_{k}= & d\left(g\left(\omega, \zeta_{l(k)}(\omega)\right), g\left(\omega, \zeta_{m(k)}(\omega)\right)\right)+d\left(g\left(\omega, \eta_{l(k)}(\omega)\right), g\left(\omega, \eta_{m(k)}(\omega)\right)\right) \\
\leq & d\left(g\left(\omega, \zeta_{l(k)}(\omega)\right), g\left(\omega, \zeta_{l(k)+1}(\omega)\right)\right)+d\left(g\left(\omega, \zeta_{l(k)+1}(\omega)\right), g\left(\omega, \zeta_{m(k)+1}(\omega)\right)\right) \\
& +d\left(g\left(\omega, \zeta_{m(k)+1}(\omega)\right), g\left(\omega, \zeta_{m(k)}(\omega)\right)\right)+d\left(g\left(\omega, \eta_{l(k)}(\omega)\right), g\left(\omega, \eta_{l(k)+1}(\omega)\right)\right) \\
& +d\left(g\left(\omega, \eta_{l(k)+1}(\omega)\right), g\left(\omega, \eta_{m(k)+1}(\omega)\right)\right)+d\left(g\left(\omega, \eta_{m(k)+1}(\omega)\right), g\left(\omega, \eta_{m(k)}(\omega)\right)\right) \\
= & d\left(g\left(\omega, \zeta_{l(k)}(\omega)\right), g\left(\omega, \zeta_{l(k)+1}(\omega)\right)\right)+d\left(g\left(\omega, \eta_{l(k)}(\omega)\right), g\left(\omega, \eta_{l(k)+1}(\omega)\right)\right) \\
& +\left[d\left(g\left(\omega, \zeta_{m(k)+1}(\omega)\right), g\left(\omega, \zeta_{m(k)}(\omega)\right)\right)+d\left(g\left(\omega, \eta_{m(k)}(\omega)\right), g\left(\omega, \eta_{m(k)+1}(\omega)\right)\right)\right] \\
& +d\left(g\left(\omega, \zeta_{l(k)+1}(\omega)\right), g\left(\omega, \zeta_{m(k)+1}(\omega)\right)\right)+d\left(g\left(\omega, \eta_{l(k)+1}(\omega)\right), g\left(\omega, \eta_{m(k)+1}(\omega)\right)\right) .
\end{aligned}
$$

Hence,

$$
\begin{aligned}
r_{k} \leq & \delta_{l(k)}+\delta_{m(k)}+d\left(g\left(\omega, \zeta_{l(k)+1}(\omega)\right), g\left(\omega, \zeta_{m(k)+1}(\omega)\right)\right) \\
& +d\left(g\left(\omega, \eta_{l(k)+1}(\omega)\right), g\left(\omega, \eta_{m(k)+1}(\omega)\right)\right) .
\end{aligned}
$$

From (5) and (6), we conclude that $g\left(\omega, \zeta_{l(k)}(\omega)\right) \leq g\left(\omega, \zeta_{m(k)}(\omega)\right)$ and $g\left(\omega, \eta_{l(k)}(\omega)\right) \geq$ $g\left(\omega, \eta_{m(k)}(\omega)\right)$.

Now (1) and (4) imply that

$$
\begin{aligned}
& d\left(g\left(\omega, \zeta_{l(k)+1}(\omega)\right), g\left(\omega, \zeta_{m(k)+1}(\omega)\right)\right) \\
& \quad=d\left(F\left(w,\left(\zeta_{l(k)}, \eta_{l(k)}(\omega)\right)\right), F\left(w,\left(\zeta_{m(k)}, \eta_{m(k)}(\omega)\right)\right)\right) \\
& \quad \leq \varphi\left(\frac{d\left(g\left(\omega, \zeta_{l(k)}(\omega)\right), g\left(\omega, \zeta_{m(k)}(\omega)\right)\right)+d\left(g\left(\omega, \eta_{l(k)}(\omega)\right), g\left(\omega, \eta_{m(k)}(\omega)\right)\right)}{2}\right) \\
& \quad=\varphi\left(\frac{r_{k}}{2}\right) .
\end{aligned}
$$

Also, from (1) and (4), as $g\left(\omega, \eta_{m(k)}(\omega)\right) \leq g\left(\omega, \eta_{l(k)}(\omega)\right)$ and $g\left(\omega, \zeta_{m(k)}(\omega)\right) \geq g\left(\omega, \zeta_{l(k)}(\omega)\right)$,

$$
\begin{aligned}
& d\left(g\left(\omega, \eta_{l(k)+1}(\omega)\right), g\left(\omega, \zeta_{m(k)+1}(\omega)\right)\right) \\
& \quad=d\left(F\left(w,\left(\eta_{l(k)}, \zeta_{l(k)}(\omega)\right)\right), F\left(w,\left(\eta_{m(k)}, \zeta_{m(k)}(\omega)\right)\right)\right) \\
& \quad \leq \varphi\left(\frac{d\left(g\left(\omega, \eta_{l(k)}(\omega)\right), g\left(\omega, \eta_{m(k)}(\omega)\right)\right)+d\left(g\left(\omega, \zeta_{l(k)}(\omega)\right), g\left(\omega, \zeta_{m(k)}(\omega)\right)\right)}{2}\right) \\
& =\varphi\left(\frac{r_{k}}{2}\right) .
\end{aligned}
$$


Inserting (18) and (19) in (17), we obtain

$$
r_{k} \leq \delta_{l(k)}+\delta_{m(k)}+2 \varphi\left(\frac{r_{k}}{2}\right)
$$

Letting $k \rightarrow \infty$, we get by $(16)$

$$
\epsilon \leq 2 \lim _{k \rightarrow \infty} \varphi\left(\frac{r_{k}}{2}\right)=2 \lim _{r_{k} \rightarrow \epsilon+} \varphi\left(\frac{r_{k}}{2}\right)<2 \frac{\epsilon}{2}=\epsilon,
$$

a contradiction. Therefore, our supposition (14) was wrong. Thus, we proved that $\left\{g\left(\omega, \zeta_{n}(\omega)\right)\right\}$ and $\left\{g\left(\omega, \eta_{n}(\omega)\right)\right\}$ are Cauchy sequences in $X$.

Since $X$ is complete and $g(\omega \times X)=X$, there exist $\zeta_{0}, \theta_{0} \in \Theta$ such that $\lim _{n \rightarrow \infty} g(\omega$, $\left.\zeta_{n}(\omega)\right)=g\left(\omega, \zeta_{0}(\omega)\right)$ and $\lim _{n \rightarrow \infty} g\left(\omega, \eta_{n}(\omega)\right)=g\left(\omega, \theta_{0}(\omega)\right)$. Since $g\left(\omega, \zeta_{0}(\omega)\right)$ and $g\left(\omega, \theta_{0}(\omega)\right)$ are measurable, therefore the functions $\zeta(\omega)$ and $\theta(\omega)$, defined by $\zeta(\omega)=g\left(\omega, \zeta_{0}(\omega)\right)$ and $\theta(\omega)=g\left(\omega, \theta_{0}(\omega)\right)$ are measurable. Thus,

$$
\begin{aligned}
& \lim _{n \rightarrow \infty} F\left(\omega,\left(\zeta_{n}(\omega), \eta_{n}(\omega)\right)\right)=\lim _{n \rightarrow \infty} g\left(\omega, \zeta_{n}(\omega)\right)=\zeta(\omega) \quad \text { and } \\
& \lim _{n \rightarrow \infty} F\left(\omega,\left(\eta_{n}(\omega), \zeta_{n}(\omega)\right)\right)=\lim _{n \rightarrow \infty} g\left(\omega, \eta_{n}(\omega)\right)=\theta(\omega) .
\end{aligned}
$$

Since $F$ and $g$ are compatible mappings, we have by (21)

$$
\begin{aligned}
& \lim _{n \rightarrow \infty} d\left(g\left(\omega, F\left(\omega,\left(\zeta_{n}(\omega), \eta_{n}(\omega)\right)\right)\right), F\left(\omega,\left(g\left(\omega, \zeta_{n}(\omega)\right), g\left(\omega, \eta_{n}(\omega)\right)\right)\right)\right)=0, \\
& \lim _{n \rightarrow \infty} d\left(g\left(\omega, F\left(\omega,\left(\eta_{n}(\omega), \zeta_{n}(\omega)\right)\right)\right), F\left(\omega,\left(g\left(\omega, \eta_{n}(\omega)\right), g\left(\omega, \zeta_{n}(\omega)\right)\right)\right)\right)=0 .
\end{aligned}
$$

Next, we prove that

$$
g(\omega, \zeta(\omega))=F(\omega,(\zeta(\omega), \theta(\omega)))
$$

and

$$
g(\omega, \theta(\omega))=F(\omega,(\theta(\omega), \zeta(\omega)))
$$

Let (a) hold. We have

$$
\begin{aligned}
& d\left(g(\omega, \zeta(\omega)), F\left(\omega,\left(g\left(\omega, \zeta_{n}(\omega)\right), g\left(\omega, \eta_{n}(\omega)\right)\right)\right)\right) \\
& \quad \leq d\left(g(\omega, \zeta(\omega)), g\left(\omega, F\left(\omega,\left(\zeta_{n}(\omega), \eta_{n}(\omega)\right)\right)\right)\right) \\
& \quad+d\left(g\left(\omega, F\left(\omega,\left(\zeta_{n}(\omega), \eta_{n}(\omega)\right)\right)\right), F\left(\omega,\left(g\left(\omega, \zeta_{n}(\omega)\right), g\left(\omega, \eta_{n}(\omega)\right)\right)\right)\right) .
\end{aligned}
$$

Taking the limit as $n \rightarrow \infty$, using (4), (21), and (22) and the fact that $F$ and $g$ are continuous, we have

$$
d(g(\omega, \zeta(\omega)), F(\omega,(\zeta(\omega), \theta(\omega))))=0
$$

Similarly, from (4), (21), and (23) and the continuity of $F$ and $g$, we have

$$
d(g(\omega, \theta(\omega)), F(\omega,(\theta(\omega), \zeta(\omega))))=0
$$


Combining the above two results, we obtain

$$
g(\omega, \zeta(\omega))=F(\omega,(\zeta(\omega), \theta(\omega)))
$$

and

$$
g(\omega, \theta(\omega))=F(\omega,(\theta(\omega), \zeta(\omega)))
$$

for each $\omega \in \Omega$.

Next, suppose that (b) holds. From (5), (6), and (21), we have $\left\{g\left(\omega, \zeta_{n}(\omega)\right)\right\}$ is nondecreasing and $\left\{g\left(\omega, \eta_{n}(\omega)\right)\right\}$ is non-increasing sequence and

$$
g\left(\omega, \zeta_{n}(\omega)\right) \rightarrow g(\omega, \zeta(\omega)), \quad g\left(\omega, \eta_{n}(\omega)\right) \rightarrow g(\omega, \theta(\omega))
$$

So, from (2) and (3), we have for all $n \geq 0$

$$
g\left(\omega, \zeta_{n}(\omega)\right) \leq g(\omega, \zeta(\omega)) \text { and } \quad g\left(\omega, \eta_{n}(\omega)\right) \geq g(\omega, \theta(\omega)) .
$$

Since $F$ and $g$ are compatible mappings and $g$ is continuous, by (22) and (23) we have

$$
\begin{aligned}
& \lim _{n \rightarrow \infty} g\left(\omega, g\left(\omega, \zeta_{n}(\omega)\right)\right) \\
& \quad=g(\omega, \zeta(\omega))=\lim _{n \rightarrow \infty} g\left(\omega, F\left(\omega,\left(\zeta_{n}(\omega), \eta_{n}(\omega)\right)\right)\right) \\
& \quad=\lim _{n \rightarrow \infty} F\left(\omega,\left(g\left(\omega, \zeta_{n}(\omega)\right), g\left(\omega, \eta_{n}(\omega)\right)\right)\right)
\end{aligned}
$$

and

$$
\begin{aligned}
& \lim _{n \rightarrow \infty} g\left(\omega, g\left(\omega, \eta_{n}(\omega)\right)\right) \\
& \quad=g(\omega, \theta(\omega))=\lim _{n \rightarrow \infty} g\left(\omega, F\left(\omega,\left(\eta_{n}(\omega), \zeta_{n}(\omega)\right)\right)\right) \\
& \quad=\lim _{n \rightarrow \infty} F\left(\omega,\left(g\left(\omega, \eta_{n}(\omega)\right), g\left(\omega, \zeta_{n}(\omega)\right)\right)\right) .
\end{aligned}
$$

Now, we have

$$
\begin{aligned}
& d(g(\omega, \zeta(\omega)), F(\omega,(\zeta(\omega), \theta(\omega)))) \\
& \quad \leq d\left(g(\omega, \zeta(\omega)), g\left(\omega, g\left(\omega, \zeta_{n+1}(\omega)\right)\right)\right) \\
& \quad+d\left(g\left(\omega, g\left(\omega, \zeta_{n+1}(\omega)\right)\right), F(\omega,(\zeta(\omega), \theta(\omega)))\right)
\end{aligned}
$$

Taking the limit as $n \rightarrow \infty$ in the above inequality, using (4) and (25), we have

$$
\begin{aligned}
& d(g(\omega, \zeta(\omega)), F(\omega,(\zeta(\omega), \theta(\omega)))) \\
& \leq \lim _{n \rightarrow \infty} d\left(g(\omega, \zeta(\omega)), g\left(\omega, g\left(\omega, \zeta_{n+1}(\omega)\right)\right)\right) \\
& \quad+\lim _{n \rightarrow \infty} d\left(g\left(\omega, F\left(\omega,\left(\zeta_{n}(\omega), \eta_{n}(\omega)\right)\right)\right), F(\omega,(\zeta(\omega), \theta(\omega)))\right) \\
& \leq \lim _{n \rightarrow \infty} d\left(F\left(\omega,\left(g\left(\omega, \zeta_{n}(\omega)\right), g\left(\omega, \eta_{n}(\omega)\right)\right)\right), F(\omega,(\zeta(\omega), \theta(\omega)))\right) .
\end{aligned}
$$


Since the mapping $g$ is monotone increasing, by (1), (24), and the above inequality, we have for all $n \geq 0$

$$
\begin{aligned}
& d(g(\omega, \zeta(\omega)), F(\omega,(\zeta(\omega), \theta(\omega)))) \\
& \quad \leq \lim _{n \rightarrow \infty} \varphi\left(\frac{d\left(g\left(\omega,\left(g\left(\omega, \zeta_{n}(\omega)\right), g(\omega, \zeta(\omega))\right)\right)\right)+d\left(g\left(\omega,\left(g\left(\omega, \eta_{n}(\omega)\right), g(\omega, \theta(\omega))\right)\right)\right)}{2}\right) .
\end{aligned}
$$

Using (21) and the property of a $\varphi$-function, we obtain

$$
d(g(\omega, \zeta(\omega)), F(\omega,(\zeta(\omega), \theta(\omega)))) \leq 0
$$

That is,

$$
g(\omega, \zeta(\omega))=F(\omega,(\zeta(\omega), \theta(\omega)))
$$

And similarly, by the virtue of (4), (21), and (26), we obtain

$$
g(\omega, \theta(\omega))=F(\omega,(\theta(\omega), \zeta(\omega))) .
$$

This proves that $F$ and $g$ have a coupled random coincidence point.

Corollary 3.3 Let $(X, \leq, d)$ be a complete separable partially ordered metric space, $(\Omega, \Sigma)$ be a measurable space, and $F: \Omega \times(X \times X) \rightarrow X$ and $g: \Omega \times X \rightarrow X$ be mappings such that

(i) $g(\omega, \cdot)$ is continuous for all $\omega \in \Omega$;

(ii) $F(\cdot, v), g(\cdot, x)$ are measurable for all $v \in X \times X$ and $x \in X$ respectively;

(iii) $F(\omega, \cdot)$ has the mixed $g(\omega, \cdot)$-monotone property for each $\omega \in \Omega$ and for some $k \in[0,1)$

$$
d(F(\omega,(x, y)), F(\omega,(u, v))) \leq \frac{k}{2}(d(g(\omega, x), g(\omega, u))+d(g(\omega, y), g(\omega, v)))
$$

for all $x, y, u, v \in X$, for which $g(\omega, x) \leq g(\omega, u)$ and $g(\omega, y) \geq g(\omega, v)$ for all $\omega \in \Omega$.

Suppose $g(\omega \times X)=X$ for each $\omega \in \Omega, g$ is monotone increasing, and $F$ and $g$ are compatible random operators. Also suppose either

(a) $F(\omega, \cdot)$ is continuous for all $\omega \in \Omega$ or

(b) $X$ has the following property:

(i) if a non-decreasing sequence $\left\{x_{n}\right\} \rightarrow x$, then $x_{n} \leq x$ for all $n$,

(ii) if a non-increasing sequence $\left\{y_{n}\right\} \rightarrow y$, then $y_{n} \geq y$ for all $n$.

If there exist measurable mappings $\zeta_{0}, \eta_{0}: \Omega \rightarrow X$ such that $g\left(\omega, \zeta_{0}(\omega)\right) \leq F(\omega$, $\left.\left(\zeta_{0}(\omega), \eta_{0}(\omega)\right)\right)$ and $g\left(\omega, \eta_{0}(\omega)\right) \geq F\left(\omega,\left(\eta_{0}(\omega), \zeta_{0}(\omega)\right)\right)$ for all $\omega \in \Omega$, then there are measurable mappings $\zeta, \theta: \Omega \rightarrow X$ such that $F(\omega, \zeta(\omega), \theta(\omega)))=g(\omega, \zeta(\omega))$ and $F(\omega,(\theta(\omega)$, $\zeta(\omega)))=g(\omega, \theta(\omega))$ for all $\omega \in \Omega$, that is, $F$ and $g$ have a coupled random coincidence point.

Proof Taking $\phi(t)=k \cdot t$ with $k \in[0,1)$ in Theorem 3.2, we obtain the result.

The following theorem presents the stochastic version of Theorem 2.7 and generalizes the recent results in [20]. 
Theorem 3.4 Let $(X, \leq, d)$ be a complete separable partially ordered metric space, $(\Omega, \Sigma)$ be a measurable space, and $F: \Omega \times(X \times X) \rightarrow X$ and $g: \Omega \times X \rightarrow X$ be mappings such that

(i) $g(\omega, \cdot)$ is continuous for all $\omega \in \Omega$;

(ii) $F(\cdot, v), g(\cdot, x)$ are measurable for all $v \in X \times X$ and $x \in X$, respectively;

(iii) $F: \Omega \times(X \times X) \rightarrow X$ and $g: \Omega \times X \rightarrow X$ are such that $F(\omega, \cdot)$ has the mixed $g(\omega, \cdot)$-monotone property for each $\omega \in \Omega$; and suppose there exist $\phi \in \Phi$ and $\psi \in \Psi$, satisfying conditions of Theorem 2.7, such that

$$
\begin{aligned}
\phi( & d(F(\omega,(x, y)), F(\omega,(u, v)))) \\
\leq & \frac{1}{2} \phi(d(g(\omega, x), g(\omega, u))+d(g(\omega, y), g(\omega, v))) \\
& -\psi\left(\frac{d(g(\omega, x), g(\omega, u))+d(g(\omega, y), g(\omega, v))}{2}\right)
\end{aligned}
$$

for all $x, y, u, v \in X$, for which $g(\omega, x) \leq g(\omega, u)$ and $g(\omega, y) \geq g(\omega, v)$ for all $\omega \in \Omega$.

Suppose $g(\omega \times X)=X$ for each $\omega \in \Omega, g$ is monotone increasing, and $F$ and $g$ are compatible random operators. Also suppose either

(a) $F(\omega, \cdot)$ is continuous for all $\omega \in \Omega$ or

(b) $X$ has the following property:

$$
\begin{aligned}
& \text { if a non-decreasing sequence }\left\{x_{n}\right\} \rightarrow X \text {, then } x_{n} \leq x \text { for all } n, \\
& \text { if a non-increasing sequence }\left\{x_{n}\right\} \rightarrow X \text {, then } x_{n} \leq x \text { for all } n \text {. }
\end{aligned}
$$

If there exist measurable mappings $\zeta_{0}, \eta_{0}: \Omega \rightarrow X$ such that $g\left(\omega, \zeta_{0}(\omega)\right) \leq F\left(\omega,\left(\zeta_{0}(\omega)\right.\right.$, $\left.\left.\eta_{0}(\omega)\right)\right)$ and $g\left(\omega, \eta_{0}(\omega)\right) \geq F\left(\omega,\left(\eta_{0}(\omega), \zeta_{0}(\omega)\right)\right)$ for all $\omega \in \Omega$, then there are measurable mappings $\zeta, \theta: \Omega \rightarrow X$ such that $F(\omega,(\zeta(\omega), \theta(\omega)))=g(\omega, \zeta(\omega))$ and $F(\omega,(\theta(\omega), \zeta(\omega)))=$ $g(\omega, \theta(\omega))$ for all $\omega \in \Omega$, that is, $F$ and $g$ have a coupled random coincidence.

Proof Let $\ominus=\{\zeta: \Omega \rightarrow X\}$ be a family of measurable mappings. Define a function $h$ : $\Omega \times X \rightarrow R^{+}$as follows:

$$
h(\omega, x)=d(x, g(\omega, x)) .
$$

Since $X \rightarrow g(\omega, x)$ is continuous for all $\omega \in \Omega$, we conclude that $h(\omega, \cdot)$ is continuous for all $\omega \in \Omega$. Also, since $x \rightarrow g(\omega, x)$ is measurable for all $x \in X$, we conclude that $h(\cdot, x)$ is measurable for all $\omega \in \Omega$ (see [26], p.868). Thus, $h(\omega, x)$ is the Caratheodory function. Therefore, if $\zeta: \Omega \rightarrow X$ is a measurable mapping, then $\omega \rightarrow h(\omega, \zeta(\omega))$ is also measurable (see [27]). Also, for each $\zeta \in \ominus$, the function $\eta: \Omega \rightarrow X$ defined by $\eta(\omega)=g(\omega, \zeta(\omega))$ is measurable, that is, $\eta \in \ominus$. Now, we shall construct two sequences of measurable mappings $\left\{\zeta_{n}\right\}$ and $\left\{\eta_{n}\right\}$ in $\ominus$, and two sequences $\left\{g\left(\omega, \zeta_{n}(\omega)\right)\right\}$ and $\left\{g\left(\omega, \eta_{n}(\omega)\right)\right\}$ in $X$ as follows. Let $\zeta_{0}, \eta_{0} \in \ominus$ such that $g\left(\omega, \zeta_{0}(\omega)\right) \leq F\left(\omega,\left(\zeta_{0}(\omega), \eta_{0}(\omega)\right)\right)$ and $g\left(\omega, \eta_{0}(\omega)\right) \geq$ $F\left(\omega,\left(\eta_{0}(\omega), \zeta_{0}(\omega)\right)\right)$ for all $\omega \in \Omega$. Since $F\left(\omega,\left(\zeta_{0}(\omega), \eta_{0}(\omega)\right)\right) \in X=g(\omega \times X)$, by an appropriate Filippov measurable implicit function theorem [1,20, 28, 29], there is $\zeta_{1} \in \ominus$ such that $g\left(\omega, \zeta_{1}(\omega)\right)=F\left(\omega,\left(\zeta_{0}(\omega), \eta_{0}(\omega)\right)\right)$. Similarly, as $F\left(\omega,\left(\eta_{0}(\omega), \zeta_{0}(\omega)\right)\right) \in X=g(\omega \times X)$, there is $\eta_{1}(\omega) \in \ominus$ such that $g\left(\omega, \eta_{1}(\omega)\right)=F\left(\omega,\left(\eta_{0}(\omega), \zeta_{0}(\omega)\right)\right)$. Now, $F\left(\omega,\left(\zeta_{1}(\omega), \eta_{1}(\omega)\right)\right)$ and 
$F\left(\omega,\left(\eta_{1}(\omega), \zeta_{1}(\omega)\right)\right)$ are well defined. Again, from $F\left(\omega,\left(\zeta_{1}(\omega), \eta_{1}(\omega)\right)\right), F\left(\omega,\left(\eta_{1}(\omega), \zeta_{1}(\omega)\right)\right) \in$ $g(\omega \times X)$, there are $\zeta_{2}, \eta_{2} \in \ominus$ such that $g\left(\omega, \zeta_{2}(\omega)\right)=F\left(\omega,\left(\zeta_{1}(\omega), \eta_{1}(\omega)\right)\right)$ and $g\left(\omega, \eta_{2}(\omega)\right)=$ $F\left(\omega,\left(\eta_{1}(\omega), \zeta_{1}(\omega)\right)\right)$. Continuing this process, we can construct sequences $\left\{\zeta_{n}(\omega)\right\}$ and $\left\{\eta_{n}(\omega)\right\}$ in $X$ such that

$$
\begin{aligned}
& g\left(\omega, \zeta_{n+1}(\omega)\right)=F\left(\omega,\left(\zeta_{n}(\omega), \eta_{n}(\omega)\right)\right) \quad \text { and } \\
& g\left(\omega, \eta_{n+1}(\omega)\right)=F\left(\omega, \eta_{n}(\omega), \zeta_{n}(\omega)\right)
\end{aligned}
$$

for all $n \geq 0$.

We shall prove that

$$
g\left(\omega, \zeta_{n}(\omega)\right) \leq g\left(\omega, \zeta_{n+1}(\omega)\right) \quad \text { for all } n \geq 0
$$

and

$$
g\left(\omega, \eta_{n}(\omega)\right) \leq g\left(\omega, \eta_{n+1}(\omega)\right) \quad \text { for all } n \geq 0 .
$$

The proof will be given by mathematical induction. Let $n=0$. By assumption, we have $g\left(\omega, \zeta_{0}(\omega)\right) \leq F\left(\omega,\left(\zeta_{0}(\omega), \eta_{0}(\omega)\right)\right)$ and $g\left(\omega, \eta_{0}(\omega)\right) \geq F\left(\omega,\left(\eta_{0}(\omega), \zeta_{0}(\omega)\right)\right)$. Since $g\left(\omega, \zeta_{1}(\omega)\right)=$ $F\left(\omega,\left(\zeta_{0}(\omega), \eta_{0}(\omega)\right)\right)$ and $g\left(\omega, \eta_{1}(\omega)\right)=F\left(\omega,\left(\eta_{0}(\omega), \zeta_{0}(\omega)\right)\right)$, we have $g\left(\omega, \zeta_{0}(\omega)\right) \leq g\left(\omega, \zeta_{1}(\omega)\right)$ and $g\left(\omega, \eta_{0}(\omega)\right) \geq g\left(\omega, \eta_{1}(\omega)\right)$. Therefore, (31) and (32) hold for $n=0$. Suppose now that (31) and (32) hold for some fixed $n \geq 0$. Then $g\left(\omega, \zeta_{n}(\omega)\right) \leq g\left(\omega, \zeta_{n+1}(\omega)\right)$ and $g\left(\omega, \eta_{n}(\omega)\right) \geq$ $g\left(\omega, \eta_{n+1}(\omega)\right)$ as $F$ is monotone $g$-non-decreasing in its first argument, from (28) and (30),

$$
\begin{aligned}
& F\left(\omega,\left(\zeta_{n}(\omega), \eta_{n}(\omega)\right)\right) \leq F\left(\omega,\left(\zeta_{n+1}(\omega), \eta_{n}(\omega)\right)\right) \text { and } \\
& F\left(\omega,\left(\eta_{n+1}(\omega), \zeta_{n}(\omega)\right)\right) \leq F\left(\omega,\left(\eta_{n}(\omega), \zeta_{n}(\omega)\right)\right) .
\end{aligned}
$$

Similarly, from (29) and (30), as $g\left(\omega, \zeta_{n}(\omega)\right) \leq g\left(\omega, \zeta_{n+1}(\omega)\right)$ and $g\left(\omega, \eta_{n}(\omega)\right) \geq g\left(\omega, \eta_{n+1}(\omega)\right)$, we have

$$
\begin{aligned}
& F\left(\omega,\left(\zeta_{n+1}(\omega), \eta_{n+1}(\omega)\right)\right) \geq F\left(\omega,\left(\zeta_{n+1}(\omega), \eta_{n}(\omega)\right)\right) \text { and } \\
& F\left(\omega,\left(\eta_{n+1}(\omega), \zeta_{n}(\omega)\right)\right) \geq F\left(\omega,\left(\eta_{n+1}(\omega), \zeta_{n+1}(\omega)\right)\right) .
\end{aligned}
$$

Now, from (30), (33), and (34), we get

$$
g\left(\omega, \zeta_{n+1}(\omega)\right) \leq g\left(\omega, \zeta_{n+2}(\omega)\right)
$$

and

$$
g\left(\omega, \eta_{n+1}(\omega)\right) \leq g\left(\omega, \eta_{n+2}(\omega)\right) .
$$

Thus, by mathematical induction we conclude that (31) and (32) hold for all $n \geq 0$.

Therefore,

$$
g\left(\omega, \zeta_{0}(\omega)\right) \leq g\left(\omega, \zeta_{1}(\omega)\right) \leq \cdots \leq g\left(\omega, \zeta_{n}(\omega)\right) \leq g\left(\omega, \zeta_{n+1}\right) \leq \cdots
$$


and

$$
g\left(\omega, \eta_{0}(\omega)\right) \geq g\left(\omega, \eta_{1}(\omega)\right) \geq \cdots \geq g\left(\omega, \eta_{n}(\omega)\right) \geq \cdots \geq g\left(\omega, \eta_{n+1}\right) \geq \cdots .
$$

Since $g\left(\omega, \zeta_{n-1}(\omega)\right) \leq g\left(\omega, \zeta_{n}(\omega)\right)$ and $g\left(\omega, \eta_{n}(\omega)\right) \leq g\left(\omega, \eta_{n-1}(\omega)\right)$, using (27) and (30), we have

$$
\begin{aligned}
\phi( & \left.d\left(g\left(\omega, \zeta_{n+1}(\omega)\right), g\left(\omega, \zeta_{n}(\omega)\right)\right)\right) \\
= & \phi\left(d\left(F\left(\omega,\left(\zeta_{n}(\omega), \eta_{n}(\omega)\right)\right)\right), F\left(\omega,\left(\zeta_{n-1}(\omega), \eta_{n-1}(\omega)\right)\right)\right) \\
\leq & \frac{1}{2} \phi\left(d\left(g\left(\omega, \zeta_{n}(\omega)\right), g\left(\omega, \zeta_{n-1}(\omega)\right)\right)+d\left(g\left(\omega, \eta_{n}(\omega)\right), g\left(\omega, \eta_{n-1}(\omega)\right)\right)\right) \\
& -\psi\left(\frac{d\left(g\left(\omega, \zeta_{n}(\omega)\right), g\left(\omega, \zeta_{n-1}(\omega)\right)\right)+d\left(g\left(\omega, \eta_{n}(\omega)\right), g\left(\omega, \eta_{n-1}(\omega)\right)\right)}{2}\right) .
\end{aligned}
$$

Similarly, since $g\left(\omega, \zeta_{n-1}(\omega)\right) \leq g\left(\omega, \zeta_{n}(\omega)\right)$ and $g\left(\omega, \eta_{n-1}(\omega)\right) \leq g\left(\omega, \eta_{n}(\omega)\right)$, using $(27)$ and (30), we also have

$$
\begin{aligned}
& \phi(\left.d\left(g\left(\omega, \eta_{n}(\omega)\right), g\left(\omega, \eta_{n+1}(\omega)\right)\right)\right) \\
&= \phi\left(d\left(F\left(\omega,\left(\eta_{n-1}(\omega), \zeta_{n-1}(\omega)\right)\right)\right), F\left(\omega,\left(\eta_{n}(\omega), \zeta_{n}(\omega)\right)\right)\right) \\
& \leq \frac{1}{2} \phi\left(d\left(g\left(\omega, \eta_{n-1}(\omega)\right), g\left(\omega, \eta_{n}(\omega)\right)\right)+d\left(g\left(\omega, \zeta_{n-1}(\omega)\right), g\left(\omega, \zeta_{n}(\omega)\right)\right)\right) \\
& \quad-\psi\left(\frac{d\left(g\left(\omega, \eta_{n-1}(\omega)\right), g\left(\omega, \eta_{n}(\omega)\right)\right)+d\left(g\left(\omega, \zeta_{n-1}(\omega)\right), g\left(\omega, \zeta_{n}(\omega)\right)\right)}{2}\right) .
\end{aligned}
$$

Using (39) and (40), we have

$$
\begin{gathered}
\phi\left(d\left(g\left(\omega, \zeta_{n+1}(\omega)\right), g\left(\omega, \zeta_{n}(\omega)\right)\right)\right)+\phi\left(d\left(g\left(\omega, \eta_{n}(\omega)\right), g\left(\omega, \eta_{n+1}(\omega)\right)\right)\right) \\
\leq \phi\left(d\left(g\left(\omega, \zeta_{n}(\omega)\right), g\left(\omega, \zeta_{n-1}(\omega)\right)\right)+d\left(g\left(\omega, \eta_{n}(\omega)\right), g\left(\omega, \eta_{n-1}(\omega)\right)\right)\right) \\
\quad-2 \psi\left(\frac{d\left(g\left(\omega, \eta_{n-1}(\omega)\right), g\left(\omega, \eta_{n}(\omega)\right)\right)+d\left(g\left(\omega, \zeta_{n-1}(\omega)\right), g\left(\omega, \zeta_{n}(\omega)\right)\right)}{2}\right)
\end{gathered}
$$

From the property (iii) of $\phi$, we have

$$
\begin{aligned}
& \phi\left(d\left(g\left(\omega, \zeta_{n+1}(\omega)\right), g\left(\omega, \zeta_{n}(\omega)\right)\right)+d\left(g\left(\omega, \eta_{n+1}(\omega)\right), g\left(\omega, \eta_{n}(\omega)\right)\right)\right) \\
& \leq \phi\left(d\left(g\left(\omega, \zeta_{n+1}(\omega)\right), g\left(\omega, \zeta_{n}(\omega)\right)\right)\right) \\
& \quad+\phi\left(d\left(g\left(\omega, \eta_{n+1}(\omega)\right), g\left(\omega, \eta_{n}(\omega)\right)\right)\right) .
\end{aligned}
$$

Using (41) and (42), we have

$$
\begin{aligned}
\phi( & \left.d\left(g\left(\omega, \zeta_{n+1}(\omega)\right), g\left(\omega, \zeta_{n}(\omega)\right)\right)+d\left(g\left(\omega, \eta_{n+1}(\omega)\right), g\left(\omega, \eta_{n}(\omega)\right)\right)\right) \\
\leq & \phi\left(d\left(g\left(\omega, \zeta_{n}(\omega)\right), g\left(\omega, \zeta_{n-1}(\omega)\right)\right)+d\left(g\left(\omega, \eta_{n}(\omega)\right), g\left(\omega, \eta_{n-1}(\omega)\right)\right)\right) \\
& -2 \psi\left(\frac{d\left(g\left(\omega, \eta_{n-1}(\omega)\right), g\left(\omega, \eta_{n}(\omega)\right)\right)+d\left(g\left(\omega, \zeta_{n-1}(\omega)\right), g\left(\omega, \zeta_{n}(\omega)\right)\right)}{2}\right)
\end{aligned}
$$


Since $\psi$ is a non-negative function, therefore we have

$$
\begin{aligned}
& \phi\left(d\left(g\left(\omega, \zeta_{n+1}(\omega)\right), g\left(\omega, \zeta_{n}(\omega)\right)\right)+d\left(g\left(\omega, \eta_{n+1}(\omega)\right), g\left(\omega, \eta_{n}(\omega)\right)\right)\right) \\
& \quad \leq \phi\left(d\left(g\left(\omega, \zeta_{n}(\omega)\right), g\left(\omega, \zeta_{n-1}(\omega)\right)\right)+d\left(g\left(\omega, \eta_{n}(\omega)\right), g\left(\omega, \eta_{n-1}(\omega)\right)\right)\right) .
\end{aligned}
$$

Using the fact that $\phi$ is non-decreasing, we get

$$
\begin{aligned}
& d\left(g\left(\omega, \zeta_{n+1}(\omega)\right), g\left(\omega, \zeta_{n}(\omega)\right)\right)+d\left(g\left(\omega, \eta_{n+1}(\omega)\right), g\left(\omega, \eta_{n}(\omega)\right)\right) \\
& \quad \leq d\left(g\left(\omega, \zeta_{n}(\omega)\right), g\left(\omega, \zeta_{n-1}(\omega)\right)\right)+d\left(g\left(\omega, \eta_{n}(\omega)\right), g\left(\omega, \eta_{n-1}(\omega)\right)\right) .
\end{aligned}
$$

Let

$$
\delta_{n}=d\left(g\left(\omega, \zeta_{n+1}(\omega)\right), g\left(\omega, \zeta_{n}(\omega)\right)\right)+d\left(g\left(\omega, \eta_{n+1}(\omega)\right), g\left(\omega, \eta_{n}(\omega)\right)\right)
$$

Now, we show that $\delta_{n} \rightarrow 0$ as $n \rightarrow \infty$. It is clear that the sequence $\left\{\delta_{n}\right\}$ is decreasing; therefore, there is some $\delta \geq 0$ such that

$$
\begin{aligned}
\lim _{n \rightarrow \infty} \delta_{n} & =\lim _{n \rightarrow \infty}\left[d\left(g\left(\omega, \zeta_{n+1}(\omega)\right), g\left(\omega, \zeta_{n}(\omega)\right)\right)+d\left(g\left(\omega, \eta_{n+1}(\omega)\right), g\left(\omega, \eta_{n}(\omega)\right)\right)\right] \\
& =\delta .
\end{aligned}
$$

We shall show that $\delta=0$. Suppose, to the contrary, that $\delta>0$. Then taking the limit as $n \rightarrow \infty$ on both sides of (43) and as $\lim _{t \rightarrow r} \psi(t)>0$ for all $r>0$ and $\phi$ is continuous, we have

$$
\begin{aligned}
\phi(\delta) & =\lim _{n \rightarrow \infty} \phi\left(\delta_{n}\right) \leq \lim _{n \rightarrow \infty}\left[\phi\left(\delta_{n-1}\right)-2 \psi\left(\frac{\delta_{n-1}}{2}\right)\right] \\
& =\phi(\delta)-2 \lim _{\delta_{n-1} \rightarrow \delta} \psi\left(\frac{\delta_{n-1}}{2}\right)<\phi(\delta),
\end{aligned}
$$

a contradiction. Thus, $\delta=0$, that is

$$
\begin{aligned}
\lim _{n \rightarrow \infty} \delta_{n} & =\lim _{n \rightarrow \infty}\left[d\left(g\left(\omega, \zeta_{n+1}(\omega)\right), g\left(\omega, \zeta_{n}(\omega)\right)\right)+d\left(g\left(\omega, \eta_{n+1}(\omega)\right), g\left(\omega, \eta_{n}(\omega)\right)\right)\right] \\
& =0
\end{aligned}
$$

Now, we will prove that $\left\{g\left(\omega, \zeta_{n}\right)\right\},\left\{g\left(\omega, \eta_{n}\right)\right\}$ are Cauchy sequences. Suppose, to the contrary, that at least one of $\left\{g\left(\omega, \zeta_{n}\right)\right\}$ or $\left\{g\left(\omega, \eta_{n}\right)\right\}$ is not a Cauchy sequence. Then there exists an $\epsilon>0$ for which we can find subsequences of positive integers $\left\{m_{k}\right\},\left\{n_{k}\right\}$ with $n(k)>m(k) \geq k$ such that

$$
\begin{aligned}
r_{k} & :=d\left(g\left(\omega, \zeta_{n(k)}(\omega)\right), g\left(\omega, \zeta_{m(k)}(\omega)\right)\right)+d\left(g\left(\omega, \eta_{n(k)}(\omega)\right), g\left(\omega, \eta_{m(k)}(\omega)\right)\right) \\
& \geq \epsilon
\end{aligned}
$$

for $k=\{1,2,3, \ldots\}$. We may also assume

$$
d\left(g\left(\omega, \zeta_{n(k)-1}(\omega)\right), g\left(\omega, \zeta_{m(k)}(\omega)\right)\right)+d\left(g\left(\omega, \eta_{n(k)-1}(\omega)\right), g\left(\omega, \eta_{m(k)}(\omega)\right)\right)<\epsilon
$$


by choosing $n(k)$ in such a way that it is the smallest integer with $n(k)>m(k)$ and satisfying (46). Using (46), (47), and the triangle inequality, we have

$$
\begin{aligned}
\epsilon \leq & r_{k}:=d\left(g\left(\omega, \zeta_{n(k)}(\omega)\right), g\left(\omega, \zeta_{m(k)}(\omega)\right)\right)+d\left(g\left(\omega, \eta_{n(k)}(\omega)\right), g\left(\omega, \eta_{m(k)}(\omega)\right)\right) \\
\leq & d\left(g\left(\omega, \zeta_{n(k)}(\omega)\right), g\left(\omega, \zeta_{n(k)-1}(\omega)\right)\right)+d\left(g\left(\omega, \zeta_{n(k)-1}(\omega)\right), g\left(\omega, \zeta_{m(k)}(\omega)\right)\right) \\
& +d\left(g\left(\omega, \eta_{n(k)}(\omega)\right), g\left(\omega, \eta_{n(k)-1}(\omega)\right)\right)+d\left(g\left(\omega, \eta_{n(k)-1}(\omega)\right), g\left(\omega, \eta_{m(k)}(\omega)\right)\right) \\
\leq & d\left(g\left(\omega, \zeta_{n(k)}(\omega)\right), g\left(\omega, \zeta_{n(k)-1}(\omega)\right)\right)+d\left(g\left(\omega, \eta_{n(k)}(\omega)\right), g\left(\omega, \eta_{n(k)-1}(\omega)\right)\right) \\
& +d\left(g\left(\omega, \zeta_{n(k)-1}(\omega)\right), g\left(\omega, \zeta_{m(k)}(\omega)\right)\right)+d\left(g\left(\omega, \eta_{n(k)-1}(\omega)\right), g\left(\omega, \eta_{m(k)}(\omega)\right)\right) \\
\leq & d\left(g\left(\omega, \zeta_{n(k)}(\omega)\right), g\left(\omega, \zeta_{n(k)-1}(\omega)\right)\right)+d\left(g\left(\omega, \eta_{n(k)}(\omega)\right), g\left(\omega, \eta_{n(k)-1}(\omega)\right)\right)+\epsilon .
\end{aligned}
$$

Letting $k \rightarrow \infty$ and using (45), we get

$$
\begin{aligned}
\lim _{k \rightarrow \infty} r_{k} & =\lim _{k \rightarrow \infty}\left[d\left(g\left(\omega, \zeta_{n(k)}(\omega)\right), g\left(\omega, \zeta_{m(k)}(\omega)\right)\right)+d\left(g\left(\omega, \eta_{n(k)}(\omega)\right), g\left(\omega, \eta_{m(k)}(\omega)\right)\right)\right] \\
& =\epsilon .
\end{aligned}
$$

By the triangle inequality,

$$
\begin{aligned}
r_{k}:= & d\left(g\left(\omega, \zeta_{n(k)}(\omega)\right), g\left(\omega, \zeta_{m(k)}(\omega)\right)\right)+d\left(g\left(\omega, \eta_{n(k)}(\omega)\right), g\left(\omega, \eta_{m(k)}(\omega)\right)\right) \\
\leq & d\left(g\left(\omega, \zeta_{n(k)}(\omega)\right), g\left(\omega, \zeta_{n(k)+1}(\omega)\right)\right)+d\left(g\left(\omega, \zeta_{n(k)+1}(\omega)\right), g\left(\omega, \zeta_{m(k)+1}(\omega)\right)\right) \\
& +d\left(g\left(\omega, \zeta_{m(k)+1}(\omega)\right), g\left(\omega, \zeta_{m(k)}(\omega)\right)\right)+d\left(g\left(\omega, \eta_{n(k)}(\omega)\right), g\left(\omega, \eta_{n(k)+1}(\omega)\right)\right) \\
& +d\left(g\left(\omega, \eta_{n(k)+1}(\omega)\right), g\left(\omega, \eta_{m(k)+1}(\omega)\right)\right)+d\left(g\left(\omega, \eta_{m(k)+1}(\omega)\right), g\left(\omega, \eta_{m(k)}(\omega)\right)\right) \\
= & d\left(g\left(\omega, \zeta_{n(k)}(\omega)\right), g\left(\omega, \zeta_{n(k)+1}(\omega)\right)\right)+d\left(g\left(\omega, \eta_{n(k)}(\omega)\right), g\left(\omega, \eta_{n(k)+1}(\omega)\right)\right) \\
& +d\left(g\left(\omega, \zeta_{m(k)+1}(\omega)\right), g\left(\omega, \zeta_{m(k)}(\omega)\right)\right)+d\left(g\left(\omega, \eta_{m(k)+1}(\omega)\right), g\left(\omega, \eta_{m(k)}(\omega)\right)\right) \\
& +d\left(g\left(\omega, \zeta_{n(k)+1}(\omega)\right), g\left(\omega, \zeta_{m(k)+1}(\omega)\right)\right)+d\left(g\left(\omega, \eta_{n(k)+1}(\omega)\right), g\left(\omega, \eta_{m(k)+1}(\omega)\right)\right), \\
r_{k}= & \delta_{n(k)}+\delta_{m(k)}+d\left(g\left(\omega, \zeta_{n(k)+1}(\omega)\right), g\left(\omega, \zeta_{m(k)+1}(\omega)\right)\right) \\
& +d\left(g\left(\omega, \eta_{n(k)+1}(\omega)\right), g\left(\omega, \eta_{m(k)+1}(\omega)\right)\right) .
\end{aligned}
$$

Using the property of $\phi$, we have

$$
\begin{aligned}
\phi\left(r_{k}\right)= & \phi\left(\delta_{n(k)}+\delta_{m(k)}+d\left(g\left(\omega, \zeta_{n(k)+1}(\omega)\right), g\left(\omega, \zeta_{m(k)+1}(\omega)\right)\right)\right. \\
& \left.+d\left(g\left(\omega, \eta_{n(k)+1}(\omega)\right), g\left(\omega, \eta_{m(k)+1}(\omega)\right)\right)\right) \\
\leq & \phi\left(\delta_{n(k)}+\delta_{m(k)}\right)+\phi\left(d\left(g\left(\omega, \zeta_{n(k)+1}(\omega)\right), g\left(\omega, \zeta_{m(k)+1}(\omega)\right)\right)\right) \\
& +\phi\left(d\left(g\left(\omega, \eta_{n(k)+1}(\omega)\right), g\left(\omega, \eta_{m(k)+1}(\omega)\right)\right)\right) .
\end{aligned}
$$

Since $n(k)>m(k)$, hence $g\left(\omega, \zeta_{n(k)}(\omega)\right) \geq g\left(\omega, \zeta_{m(k)}(\omega)\right)$ and $g\left(\omega, \eta_{n(k)}(\omega)\right) \leq g\left(\omega, \eta_{m(k)}(\omega)\right)$. Using (27) and (30), we get

$$
\begin{aligned}
\phi( & \left.d\left(g\left(\omega, \zeta_{n(k)+1}(\omega)\right), g\left(\omega, \zeta_{m(k)+1}(\omega)\right)\right)\right) \\
\quad & \phi\left(d\left(F\left(\omega,\left(\zeta_{n(k)}(\omega), \eta_{n(k)}(\omega)\right)\right), F\left(\omega,\left(\zeta_{m(k)}(\omega), \eta_{m(k)}(\omega)\right)\right)\right)\right)
\end{aligned}
$$




$$
\begin{aligned}
\leq & \frac{1}{2} \phi\left(d\left(g\left(\omega, \zeta_{n(k)}(\omega)\right), g\left(\omega, \zeta_{m(k)}(\omega)\right)\right)+d\left(g\left(\omega, \eta_{n(k)}(\omega)\right), g\left(\omega, \eta_{m(k)}(\omega)\right)\right)\right) \\
& -\psi\left(\frac{d\left(g\left(\omega, \zeta_{n(k)}(\omega)\right), g\left(\omega, \zeta_{m(k)}(\omega)\right)\right)+d\left(g\left(\omega, \eta_{n(k)}(\omega)\right), g\left(\omega, \eta_{m(k)}(\omega)\right)\right)}{2}\right) \\
= & \frac{1}{2} \phi\left(r_{k}\right)-\psi\left(\frac{r_{k}}{2}\right) .
\end{aligned}
$$

By the same way, we also have

$$
\begin{aligned}
\phi & \left(d\left(g\left(\omega, \eta_{n(k)+1}(\omega)\right), g\left(\omega, \eta_{m(k)+1}(\omega)\right)\right)\right) \\
= & \phi\left(d\left(F\left(\omega,\left(\eta_{n(k)}(\omega), \zeta_{n(k)}(\omega)\right)\right), F\left(\omega,\left(\eta_{m(k)}(\omega), \zeta_{m(k)}(\omega)\right)\right)\right)\right) \\
\leq & \frac{1}{2} \phi\left(d\left(g\left(\omega, \eta_{n(k)}(\omega)\right), g\left(\omega, \eta_{m(k)}(\omega)\right)\right)+d\left(g\left(\omega, \zeta_{n(k)}(\omega)\right), g\left(\omega, \zeta_{m(k)}(\omega)\right)\right)\right) \\
& -\psi\left(\frac{d\left(g\left(\omega, \eta_{n(k)}(\omega)\right), g\left(\omega, \eta_{m(k)}(\omega)\right)\right)+d\left(g\left(\omega, \zeta_{n(k)}(\omega)\right), g\left(\omega, \zeta_{m(k)}(\omega)\right)\right)}{2}\right) \\
= & \frac{1}{2} \phi\left(r_{k}\right)-\psi\left(\frac{r_{k}}{2}\right) .
\end{aligned}
$$

Putting (50) and (51) in (49), we have

$$
\begin{aligned}
\phi\left(r_{k}\right) & \leq \phi\left(\delta_{n(k)}+\delta_{m(k)}\right)+\frac{1}{2} \phi\left(r_{k}\right)-\psi\left(\frac{r_{k}}{2}\right)+\frac{1}{2} \phi\left(r_{k}\right)-\psi\left(\frac{r_{k}}{2}\right) \\
& =\phi\left(\delta_{n(k)}+\delta_{m(k)}\right)+\phi\left(r_{k}\right)-2 \psi\left(\frac{r_{k}}{2}\right) .
\end{aligned}
$$

Taking $k \rightarrow \infty$ and using (45) and (48), we get

$$
\begin{aligned}
\phi(\epsilon) & \leq \phi(0)+\phi(\epsilon)-2 \lim _{k \rightarrow \infty} \psi\left(\frac{r_{k}}{2}\right) \\
& =\phi(\epsilon)-2 \lim _{r_{k} \rightarrow \epsilon} \psi\left(\frac{r_{k}}{2}\right)<\phi(\epsilon),
\end{aligned}
$$

a contradiction. This shows that $\left\{g\left(\omega, \zeta_{n}\right)\right\}$ and $\left\{g\left(\omega, \eta_{n}\right)\right\}$ are Cauchy sequences.

Since $X$ is complete and $g(\omega \times X)=X$, there exist $\zeta_{0}, \theta_{0} \in \Theta$ such that $\lim _{n \rightarrow \infty} g(\omega$, $\left.\zeta_{n}(\omega)\right)=g\left(\omega, \zeta_{0}(\omega)\right)$ and $\lim _{n \rightarrow \infty} g\left(\omega, \eta_{n}(\omega)\right)=g\left(\omega, \theta_{0}(\omega)\right)$. Since $g\left(\omega, \zeta_{0}(\omega)\right)$ and $g\left(\omega, \theta_{0}(\omega)\right)$ are measurable, then the functions $\zeta(\omega)$ and $\theta(\omega)$, defined by $\zeta(\omega)=g\left(\omega, \zeta_{0}(\omega)\right)$ and $\theta(\omega)=g\left(\omega, \theta_{0}(\omega)\right)$, are measurable. Thus,

$$
\begin{aligned}
& \lim _{n \rightarrow \infty} F\left(\omega,\left(\zeta_{n}(\omega), \eta_{n}(\omega)\right)\right)=\lim _{n \rightarrow \infty} g\left(\omega, \zeta_{n}(\omega)\right)=\zeta(\omega) \text { and } \\
& \lim _{n \rightarrow \infty} F\left(\omega,\left(\eta_{n}(\omega), \zeta_{n}(\omega)\right)\right)=\lim _{n \rightarrow \infty} g\left(\omega, \eta_{n}(\omega)\right)=\theta(\omega) .
\end{aligned}
$$

Using the compatibility of $F$ and $g$ and the technique of the proof of Theorem 3.2, we obtain the required conclusion.

Corollary 3.5 Let $(X, \leq, d)$ be a complete separable partially ordered metric space, $(\Omega, \Sigma)$ be a measurable space, and $F: \Omega \times(X \times X) \rightarrow X$ and $g: \Omega \times X \rightarrow X$ be mappings such that 
(i) $g(\omega, \cdot)$ is continuous for all $\omega \in \Omega$;

(ii) $F(\cdot, v), g(\cdot, x)$ are measurable for all $v \in X \times X$ and $x \in X$ respectively;

(iii) $F(\omega, \cdot)$ has the mixed $g(\omega, \cdot)$-monotone property for each $\omega \in \Omega$; and suppose there exist $\phi \in \Phi$ and $\psi \in \Psi$ such that

$$
\begin{aligned}
d(F(\omega,(x, y)), F(\omega,(u, v))) \leq & \frac{1}{2}(d(g(\omega, x), g(\omega, u))+d(g(\omega, y), g(\omega, v))) \\
& -\psi\left(\frac{d(g(\omega, x), g(\omega, u))+d(g(\omega, y), g(\omega, v))}{2}\right)
\end{aligned}
$$

for all $x, y, u, v \in X$, for which $g(\omega, x) \leq g(\omega, u)$ and $g(\omega, y) \geq g(\omega, v)$ for all $\omega \in \Omega$.

Suppose $g(\omega \times X)=X$ for each $\omega \in \Omega, g$ is continuous and monotone increasing, and $F$ and $g$ are compatible mappings. Also suppose either

(a) $F(\omega, \cdot)$ is continuous for all $\omega \in \Omega$ or

(b) $X$ has the following property:

(i) if a non-decreasing sequence $\left\{x_{n}\right\} \rightarrow X$, then $x_{n} \leq x$ for all $n$,

(ii) if a non-increasing sequence $\left\{x_{n}\right\} \rightarrow X$, then $x_{n} \leq x$ for all $n$.

If there exist measurable mappings $\zeta_{0}, \eta_{0}: \Omega \rightarrow X$ such that $g\left(\omega, \zeta_{0}(\omega)\right) \leq F\left(\omega,\left(\zeta_{0}(\omega)\right.\right.$, $\left.\left.\eta_{0}(\omega)\right)\right)$ and $g\left(\omega, \eta_{0}(\omega)\right) \geq F\left(\omega,\left(\eta_{0}(\omega), \zeta_{0}(\omega)\right)\right)$ for all $\omega \in \Omega$, then there are measurable mappings $\zeta, \theta: \Omega \rightarrow X$ such that $F(\omega,(\zeta(\omega), \theta(\omega)))=g(\omega, \zeta(\omega))$ and $F(\omega,(\theta(\omega), \zeta(\omega)))=$ $g(\omega, \theta(\omega))$ for all $\omega \in \Omega$, that is, $F$ and $g$ have a coupled random coincidence.

Proof Take $\phi(t)=t$ in Theorem 3.4.

Corollary 3.6 Let $(X, \leq, d)$ be a complete separable partially ordered metric space, $(\Omega, \Sigma)$ be a measurable space, and $F: \Omega \times(X \times X) \rightarrow X$ and $g: \Omega \times X \rightarrow X$ be mappings such that

(i) $g(\omega, \cdot)$ is continuous for all $\omega \in \Omega$;

(ii) $F(\cdot, v), g(\cdot, x)$ are measurable for all $v \in X \times X$ and $x \in X$ respectively;

(iii) $F(\omega, \cdot)$ has the mixed $g(\omega, \cdot)$-monotone property for each $\omega \in \Omega$; and suppose there exists $k \in[0,1)$ such that

$$
(d(F(\omega,(x, y)), F(\omega,(u, v)))) \leq \frac{k}{2}(d(g(\omega, x), g(\omega, u))+d(g(\omega, y), g(\omega, v)))
$$

for all $x, y, u, v \in X$, for which $g(\omega, x) \leq g(\omega, u)$ and $g(\omega, y) \geq g(\omega, v)$ for all $\omega \in \Omega$.

Suppose $g(\omega \times X)=X$ for each $\omega \in \Omega, g$ is monotone increasing, and $F$ and $g$ are compatible random operators. Also suppose either

(a) $F(\omega, \cdot)$ is continuous for all $\omega \in \Omega$ or

(b) $X$ has the following property:

(i) if a non-decreasing sequence $\left\{x_{n}\right\} \rightarrow X$, then $x_{n} \leq x$ for all $n$,

(ii) if a non-increasing sequence $\left\{x_{n}\right\} \rightarrow X$, then $x_{n} \leq x$ for all $n$.

If there exist measurable mappings $\zeta_{0}, \eta_{0}: \Omega \rightarrow X$ such that $g\left(\omega, \zeta_{0}(\omega)\right) \leq F\left(\omega,\left(\zeta_{0}(\omega)\right.\right.$, $\left.\left.\eta_{0}(\omega)\right)\right)$ and $g\left(\omega, \eta_{0}(\omega)\right) \geq F\left(\omega,\left(\eta_{0}(\omega), \zeta_{0}(\omega)\right)\right)$ for all $\omega \in \Omega$, then there are measurable mappings $\zeta, \theta: \Omega \rightarrow X$ such that $F(\omega,(\zeta(\omega), \theta(\omega)))=g(\omega, \zeta(\omega))$ and $F(\omega,(\theta(\omega), \zeta(\omega)))=$ $g(\omega, \theta(\omega))$ for all $\omega \in \Omega$, that is, $F$ and $g$ have a coupled random coincidence point.

Proof Take $\psi(t)=\frac{1-k}{2} t$ in Corollary 3.5. 
Remark 3.7 By defining $g: \Omega \times X \rightarrow X$ as $g(\omega, x)=x$ for all $\omega \in \Omega$ in Theorem 3.2Corollary 3.6, we obtain corresponding coupled random fixed point results.

\section{Competing interests}

The authors declare that they have no competing interests.

\section{Authors' contributions}

All authors contributed equally and significantly in writing this article. All authors read and approved the final manuscript.

\section{Author details}

'Department of Mathematics, King Abdulaziz University, P.O. Box 80203, Jeddah, 21589, Saudi Arabia. ${ }^{2}$ Centre for Advanced Studies in Pure and Applied Mathematics, Bahauddin Zakariya University, Multan, 60800, Pakistan.

\section{Acknowledgements}

The first and second author gratefully acknowledge the support from the Deanship of Scientific Research (DSR) at King Abdulaziz University (KAU) during this research.

Received: 16 May 2012 Accepted: 16 October 2012 Published: 1 November 2012

\section{References}

1. Itoh, S: A random fixed point theorem for a multi-valued contraction mapping. Pac. J. Math. 68, 85-90 (1977)

2. Lin, TC: Random approximations and random fixed point theorems for non-self maps. Proc. Am. Math. Soc. 103, 1129-1135 (1988)

3. Abbas, $M$, Hussain, $N$, Rhoades, BE: Coincidence point theorems for multivalued $f$-weak contraction mappings and applications. Rev. R. Acad. Cienc. Exactas Fis. Nat. Ser. A, Mat. 105, 261-272 (2011)

4. Agarwal, RP, O'Regan, D, Sambandham, M: Random and deterministic fixed point theory for generalized contractive maps. Appl. Anal. 83, 711-725 (2004)

5. Beg, I, Khan, AR, Hussain, N: Approximation of *-nonexpansive random multivalued operators on Banach spaces. J. Aust. Math. Soc. 76, 51-66 (2004)

6. Ćirić, LB, Ješić, SN, Ume, JS: On random coincidence for a pair of measurable mappings. J. Inequal. Appl. 2006, Article ID 81045 (2006)

7. Huang, NJ: A principle of randomization of coincidence points with applications. Appl. Math. Lett. 12, 107-113 (1999)

8. Khan, AR, Hussain, N: Random coincidence point theorem in Frechet spaces with applications. Stoch. Anal. Appl. 22, 155-167 (2004)

9. Sehgal, VM, Singh, SP: On random approximations and a random fixed point theorem for set valued mappings. Proc. Am. Math. Soc. 95, 91-94 (1985)

10. Agarwal, RP, Hussain, N, Taoudi, MA: Fixed point theorems in ordered Banach spaces and applications to nonlinear integral equations. Abstr. Appl. Anal. 2012, Article ID 245872 (2012)

11. Chen, Y-Z: Fixed points for discontinuous monotone operators. J. Math. Anal. Appl. 291, 282-291 (2004)

12. Hussain, N, Khan, AR, Agarwal, RP: Krasnosel'skii and Ky Fan type fixed point theorems in ordered Banach spaces. J. Nonlinear Convex Anal. 11(3), 475-489 (2010)

13. Agarwal, RP, El-Gebeily, MA, O’Regan, D: Generalized contractions in partially ordered metric spaces. Appl. Anal. 87, 109-116 (2008)

14. Hussain, N, Alotaibi, A: Coupled coincidences for multi-valued nonlinear contractions in partially ordered metric spaces. Fixed Point Theory Appl. 2011, 82 (2011). doi:10.1186/1687-1812-2011-82

15. Hussain, N, Latif, A, Shah, MH: Coupled and tripled coincidence point results without compatibility. Fixed Point Theory Appl. 2012, 77 (2012)

16. Lakshimkantham, V, Vatsala, AS: General uniqueness and monotone iterative technique for fractional differential equations. Appl. Math. Lett. 21, 828-834 (2008)

17. Luong, NV, Thuan, NX: Coupled fixed point in partially ordered metric spaces and applications. Nonlinear Anal. 74 983-992 (2011)

18. Nieto, JJ, Rodriquez-Lopez, R: Contractive mapping theorems in partially ordered sets and applications to ordinary differential equations. Order 22, 223-239 (2005)

19. Ran, ACM, Reurings, MCB: A fixed point theorem in partially ordered sets and some applications to matrix equations. Proc. Am. Math. Soc. 132, 1435-1443 (2004)

20. Ćirić, LB, Lakshmikantham, V: Coupled random fixed point theorems for nonlinear contractions in partially ordered metric spaces. Stoch. Anal. Appl. 27, 1246-1259 (2009)

21. Zhu, X-H, Xiao, J-Z: Random periodic point and fixed point results for random monotone mappings in ordered Polish spaces. Fixed Point Theory Appl. 2010, Article ID 723216 (2010)

22. Alotaibi, A, Alsulami, S: Coupled coincidence points for monotone operators in partially ordered metric spaces. Fixed Point Theory Appl. 2011, 44 (2011)

23. Choudhury, BS, Kundu, A: A coupled coincidence point in partially ordered metric spaces for compatible mappings. Nonlinear Anal. 73, 2524-2531 (2010)

24. Lakshimkantham, V, Ćirić, LB: Coupled fixed point theorems for nonlinear contractions in partially ordered metric spaces. Nonlinear Anal. 70, 4341-4349 (2009)

25. Bhaskar, TG, Lakshmikantham, V: Fixed point theorems in partially ordered metric spaces and applications. Nonlinear Anal. 65, 1379-1393 (2006)

26. Wagner, DH: Survey of measurable selection theorems. SIAM J. Control Optim. 15, 859-903 (1977) 
27. Rockafellar, RT: Measurable dependence of convex sets and functions in parameters. J. Math. Anal. Appl. 28, 4-25 (1969)

28. Himmelberg, CJ: Measurable relations. Fundam. Math. 87, 53-72 (1975)

29. McShane, EJ, Warified, RB Jr.: On Filippov's implicit functions lemma. Proc. Am. Math. Soc. 18, 41-47 (1967)

doi:10.1186/1029-242X-2012-257

Cite this article as: Hussain et al.: Weak contractive inequalities and compatible mixed monotone random operators

in ordered metric spaces. Journal of Inequalities and Applications 2012 2012:257.

Submit your manuscript to a SpringerOpen ${ }^{\odot}$ journal and benefit from:

- Convenient online submission

- Rigorous peer review

- Immediate publication on acceptance

- Open access: articles freely available online

- High visibility within the field

- Retaining the copyright to your article

Submit your next manuscript at $>$ springeropen.com 